\title{
Fundamental and Second Harmonic Bands of Solar Radio Burst Type II Caused by X1.8 - Class Solar Flares
}

\author{
Z. S. Hamidi ${ }^{1, *}$, N. N. M. Shariff ${ }^{2}$, C. Monstein ${ }^{3}$ \\ ${ }^{1}$ School of Physics and Material Sciences, Faculty of Sciences, MARA University of Technology, \\ 40450, Shah Alam, Selangor, Malaysia \\ ${ }^{2}$ Academy of Contemporary Islamic Studies (ACIS), MARA University of Technology, \\ 40450, Shah Alam, Selangor, Malaysia \\ ${ }^{3}$ Institute of Astronomy, Wolfgang-Pauli-Strasse 27, Building HIT, Floor J, \\ $\mathrm{CH}-8093$ Zurich, Switzerland \\ *E-mail address: zetysh@salam.uitm.edu.my
}

\begin{abstract}
An extreme 2012 October 23 solar flare event marked on the onset of the CALLISTO data, being one of the highest solar flare event that successfully detected. The formation of harmonic solar burst type II in meter region and their associated with X1.8-class solar flares has been reported. This burst has been observed at the National Space Centre, Banting, detected by the Compound Astronomical Low-cost Low-frequency Instrument for Spectroscopy and Transportable Observatory (CALLISTO) system in the range of $150-400 \mathrm{MHz}$ in the low frequency band. It occurred between 3.17:45 UT to 3.19:00 UT within 1 minute 15 seconds. The Compound Astronomical Low-cost Low-frequency Instrument for Spectroscopy and Transportable Observatory CALLISTO spectrometer is a solar dedicated spectrometer system that has been installed all over the world to monitor the Sun activity in 24 hours. The growth of this burst is often accompanied by abundance enhancement of particles which may take the form of multiple independent drifting bands or other forms of fine structure. Due to the results, the drift rate of this burst is $2.116 \mathrm{MHz} \mathrm{s}^{-1}$, which is considered as a slow drift rate. These drifting bands are approximately having a frequency ratio $2: 1$. This burst is a particular interest, though of sporadic and infrequent occurrence. The splitting is due to the effect of magnetic splitting, analogous to the Zeeman Effect. This is one of the examples which the type II burst is not always associated with CMEs event. The combination of radio and $\mathrm{x}$-ray region give a complete view of the solar flare eruption from e active region AR1598. Both different electromagnetic spectrum shows the exact time. Other interesting results is that this type II burst is not associated with CMEs as usual, but due to the very high solar flare event with a fundamental form at more than $100 \mathrm{MHz}$. An extension of the present work will be a detailed study of the possible triggering and the driving mechanism of solar flare explosion.
\end{abstract}

Keywords: Solar physics; radio burst; type II; Zeeman Effect; solar flare; CALLISTO 


\section{INTRODUCTION}

The discovery of solar radio waves has added more than seven decades, the wavelength range from 8 millimeters to 15 meters and known as a solar spectrum. This spectrum contains a signal called a solar burst. The bursts are considered to be a significant characteristic of solar activity because they are generally attributed to a sudden acceleration of particles from the Sun $[1,2]$. Until now, the mystery of the mechanism of origin of certain solar radio waves is not easy to be understood and also stimulated a new field of theoretical research concerned with the generation of radio waves in ionized gas; and the exciting technical requirements of the observations have led to important advances in radio techniques.

It is well known that a high energy and complicated phenomenon in which mass eruptions occur, energetic particles are generated and highly energy radiations are emitted is recognized as a solar flare. The eruption mechanism of solar flares and type III are currently an extremely active area of research, especially during the solar cycle is towards maximum [3]. In this phenomenon, large quantities of energy are transferred between the corona and chromosphere through thermal conduction, non-thermal particle beams, radiation transport and mass motions. It is well known that the magnetic energy in the solar corona is explosively released before converted into the thermal and kinetic energy in solar flares [4].

This event is triggered by fast drift of individual sunspot proper motion within the complex magnetic configuration due to instabilities of equilibrium of coronal magnetic field [5]. In a previous study, we report the results of the dynamical structure of solar flare type III that occurred on 9th March 2012 at National Space Centre, Sg Lang, Selangor, Malaysia by using the CALLISTO system. An inverted U type is occurring on 9th March 2012 between 4:00 UT to 4:15 UT within 1 minute $(4: 12-4: 13)$ UT also related to high solar flare [6]. The study of solar radio burst type III is of paramount importance because of the fact that it helps to gain an insight of generation mechanisms of solar flare and Coronal Mass Ejections (CMEs) phenomena [7].

An analysis of the correlation between radio flux strength measurement and solar flare in the X-ray region has been done in the previous work [8]. These bursts are associated with solar flare type M6 [9]. The solar monitoring is one of the radio astronomy research and outreach in Malaysia [10]. As equatorial country, the characteristic features of the climate of Malaysia are uniform temperature, very high humidity and copious rainfall. Malaysia has an average of temperature of $26.7^{\circ} \mathrm{C}$ per year [11]. We believed that the mechanism of evolution the bursts play an important role to find out the factors that initiate the event [12]. The next section will discuss the evolution of solar burst type II and the following section will focus in detail the methodology, results and conclusion.

\section{SOLAR RADIO BURST TYPE II}

Solar radio burst type II was first identified by Payne Scott [13] and also discovered by Boischot [14] and classified as a broadband lasting from 20 minutes to a few hours. In general, the formation of solar radio burst type II is due to electrons accelerated at an outward propagating coronal shock front [15]. One main characteristic of this type is that it is the earliest indicators of CME-driven shocks or a very high solar flare event [16]. During 15th April 2012, solar filament eruption was accompanied by intense CMEs. This explosion of CMEs observed by the Solar Dynamics Observatory with sunspot AR1458 is crackling with C- 
class solar flares [17]. It is found that the indication of signal potentially drives Coronal Mass Ejections (CMEs) [18].

The onset time of this type precludes the possibility of the CME driven shock causing it [19]. The motion of the shock through the radial plasma density profile can be observed based on the decreasing of the signal in frequency. One can deduce the propagation speed of the driving shock wave from eruption region. Thus the CME kinetic energy is the indicator of the lifetime of the type II bursts [20]. This burst usually distinguishes from the fast-drifting type III bursts and could be produced by synchrotron radiation by electrons spiraling in a magnetic field. It exhibits considerable individual variation discovered in the dynamic spectra, as slowly drifting bands, often in pairs differing in frequency by a factor $\approx 2$ [21].

The burst was quickly interpreted in terms of a coronal shock wave accelerating electrons, driving Langmuir waves near the electron plasma frequency $f_{p}$, and producing radio emission near $f_{p}$ and $2 f_{p}$. The slope of this burst, $(d f / d t)$ can be converted to the shock speed if the density scale height $\mathrm{H}=[\mathrm{n}-1 \mathrm{dn} / \mathrm{dr}]^{-1}$ in the ambient medium is known [22]. The main band is split into two sub-bands because of the effect of magnetic splitting, analogous to the Zeeman effect. Therefore the slowly drifting feature in dynamic spectrum can be observed. It's also widely known that the radio emission it occurs as a final step in a series of physical processes: initiation of the shock, particle acceleration, generation of plasma waves, and finally conversion of plasma waves into the electromagnetic waves.

The shocks basically formed when the seed of the driver exceeds the wave propagation speed in the ambient medium called Alfven in which is governed by both the magnetic field and plasma density. This origin of metric Solar radio burst type II is still under debate. Magnetic splitting in some cases require unacceptably high speeds relative to the ions in a laminar shock model. There are two alternative models: emission from different parts in the shock front, or emission from in front and behind the shock front. In bandwidth of meter. This burst II varies from $50-300 \mathrm{MHz}$ and the bandwidth of m-type II occurs mostly at $50 \mathrm{MHz}$. It should be noted that if the emission is at the fundamental of the plasma frequency,

$$
f[M H z]=f_{p}=9 \times 10^{-3} \sqrt{n(r)}
$$

From electron density n(r), the slope of the type II burst $(d f / d t)$ can convert to the shock speed if when the density scale height $H=\left[n^{-1} d n / d r\right]^{-1}$ in the ambient medium [23] is known. This type also has a high-frequency cutoff at $\sim 150 \mathrm{MHz}$, attributed to the fact that the Alfven speed is very high in the core of the active regions and falls off rapidly as one goes away from the active region radially as well as laterally [24]. Roughly, $60 \%$ of the fast CMEs were not associated with DH type II bursts, and is expected to be radio-rich [25]. In addition, almost 93 $\%$ of the metric type II bursts did not have IP signatures based on data from November 1994 to June 1998 [26].

The impact of solar activities indirectly affected the conditions of earth's climate and space weather in general [27].

\section{METHODOLOGY AND OBSERVATION}

Radio observatories normally are located at a very minimum of population density distribution [28]. The Compound Astronomical Low-cost Low-frequency Instrument for Spectroscopy and Transportable Observatory CALLISTO spectrometer is a solar dedicated 
spectrometer system that has been installed all over the world to monitor the Sun activity in 24 hours.

There are more than 35 sites around the world that monitor the Sun activity within 24 hours until 2014 [29]. Most of the observations available consist of simple time records of the flux density of the Sun at particular wavelengths. Several series of observation have been taken using a 5.5 meters of Log Periodic Dipole Antenna (LPDA) located the National Space Centre (ANGKASA) building at the Sg. Lang, Banting, Selangor with coordinate (N 02 ${ }^{\circ} 49.488^{\prime} \mathrm{E}$ $101^{\circ} 36.168^{\prime}$ ) with the operating of frequency from $85-470 \mathrm{MHz}$ [30-32].

The gain of the antenna is $9.3 \mathrm{~dB}$ [31]. Different altitude of observation, for example, it can effect on RFI level or the noise environment to radio astronomy [33]. In order to make sure that the data of solar monitoring in radio region is reliable, we need to study the variation of interference and the possible sources that contribute to this interference [34]. This antenna is connected to the spectrometer via cable RG 58 and the modification, calibration process and basic testing of the antenna has been done in order to improve the quality of the system [35]. From the results, we found that the effective temperature of the antenna is $1.3 \mathrm{~dB}$ [36] and the temperature of the Sun during that observation is $1578 \mathrm{~K}$ [37]. To overcome the limitation of the gain,we used a preamplifier.

The data are automatically saved in FIT files. Due to constrain of the interference factors, the range of $150-350 \mathrm{MHz}$ had been chosen as selected data [38]. Radio Frequency Interference (RFI) is a major obstacle when performing observation with CALLISTO [39].The RFI from the solar burst extinguish from the Sun's corona atmosphere and it dynamical structure of the magnetic field in radio wavelength are studied [40]. The analysis is done by classifying and characterizing the strength of the sources' RFI profiles [41]. Solar monitoring is observed within 12 hours at our site daily [42]. Sunspot data are taken from Solar Influences Data Analysis Center (SIDC) [43-51]. The next section will begin to discuss the particular phenomena.

\section{RESULT AND DISCUSSION}

In our case, a fundamental and second harmonic band solar burst type II has been detected from $150 \mathrm{MHz}$ till $400 \mathrm{MHz}$ in the low frequency band occurred on 23rd October 2012 between 3:17:45 UT to 3:19:00 UT within 1 minute 15 seconds. In specific, a second harmonic structure of solar radio burst type II has been observed with maximum emission near the frequency $350 \mathrm{MHz}$. At the same time, a group and continuum type III burst can be seen behind this burst.

This burst is often accompanied by a abundance which may take the form of numerous independent drifting bands or other forms of fine structure. The main band is split into two sub-bands because of the Zeeman Effect; splitting a spectral line into several components in the presence of a static magnetic field. A band splitting of the order of $10 \mathrm{MHz}$ can also be seen in the harmonic backbone at times around 3:17:45UT to 3:18:45 UT with the peak of flux exceeds up to $620 \mathrm{sfu}$. This splitting is associated with the rising phase of the flare.

We have compared with solar flare from Geostationary Operational Environmental Satellites (GOES15) data. From the analysis, this burst is ejected and appears at the same point of the X-1.8 class solar flare event.

There are three solar active regions AR 1591, AR 1593, AR1596 and AR 1598 with the number of sunspots exceeding 86 . 


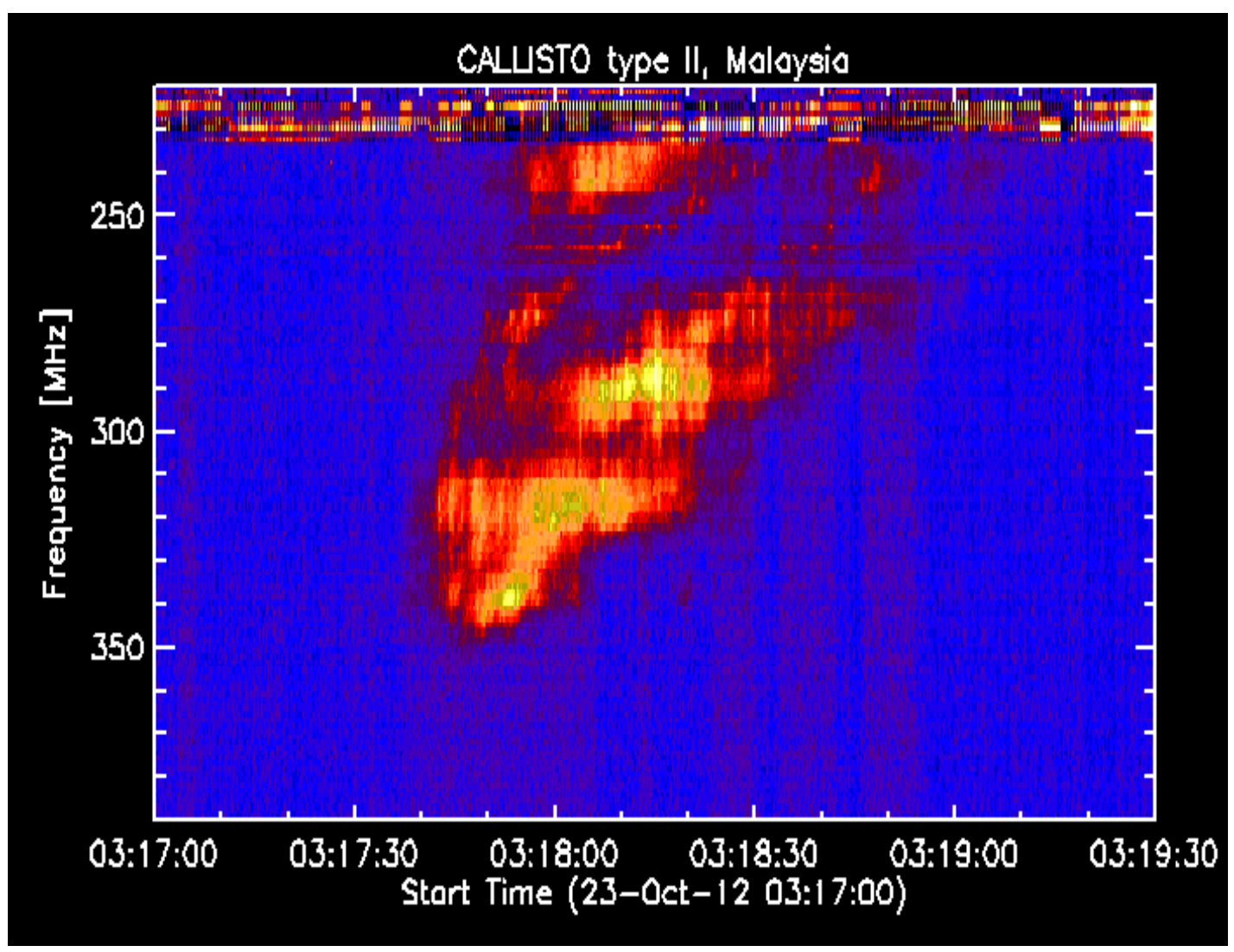

Figure 1. Type II burst with band splitting of fundamental and harmonic emission on $23^{\text {rd }}$ October 2012.

The broad features of solar radio waves are illustrated diagrammatically in Figure. 1 in terms of single-frequency records of flux density versus time. Morphologically, it is clearly observed a group and individual solar radio burst type III before the formation of solar radio burst type II. Based on the analysis, the drift rate of this burst is $2.116 \mathrm{MHz} \mathrm{s}{ }^{-1}$, which is considered as a slow drift rate. These drifting bands are approximately having a frequency ratio 2:1. The burst also accompanied by a group of solar radio burst type III since 00:00 UT. It means that it takes almost 3 hours and 17 minutes to form solar radio burst type II. The duration of the formation of the burst is long lasting until 3 minutes. The times profiles of data X-ray of solar flare for the period 22:00 UT to 4:00 UT are plotted in Figure 2 from 22:00 UT to $4: 00 \mathrm{UT}$.

Significant results of the flaring becoming more intense beginning from 3:00 UT were also determined. The highest peak of the solar flare can be observed during that time. Despite this, the bursts show wide varieties of dynamic spectra, probably because of the solar plasma inhomogeneity through which parts of the shock wave travel. The simplest is sharp-featured and continue their curved sweep towards decreasing frequencies as far as the lower frequencies observable, their duration increasing with decreasing frequency. There is also an extreme ultraviolet flash observed by the Solar Dynamics Observatory (SDO). The radiation from the solar flare created waves of ionization in the upper atmosphere over Asia and Australia and possibly HF radio blackouts at high latitudes. The blast did not, however, produce a significant Coronal Mass Ejections (CMEs). 


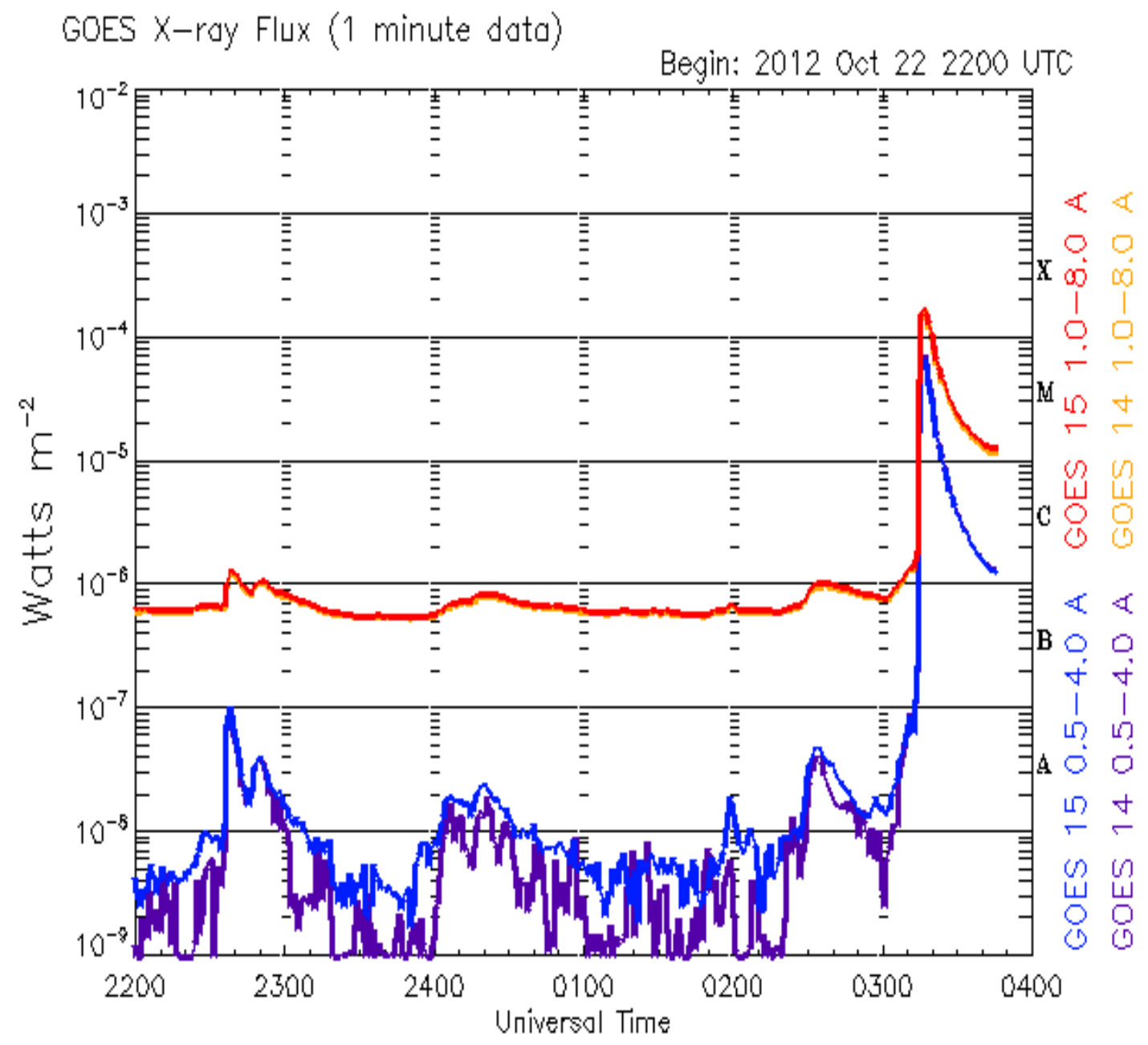

Updated 2012 oct 230347 UTC

NOAA/SWPC Boulder, CO USA

Figure 2. Type II burst with the band splitting of fundamental and harmonic emission on 23rd October 2012 (Credited to: Geostationary Operational Environmental Satellites (GOES)).

\section{CONCLUSION}

This burst is a particular interest, though of sporadic and infrequent occurrence. The burst is one of the examples where the type II burst is not always associated with CMEs event. The combination of radio and $\mathrm{x}$-ray region give a complete view of the solar flare eruption from e active region AR1598. Both different electromagnetic spectrum shows the exact time. Other interesting results is that this type II burst is not associated with CMEs as usual, but due to the very high solar flare event with a fundamental formed at more than $100 \mathrm{MHz}$. An extension of the present work will be a detailed study of the possible triggering and the driving mechanism of solar flare explosion.

\section{Acknowledgement}

We are grateful to CALLISTO network, STEREO, LASCO,SDO/AIA, NOAA and SWPC make their data available online. This work was partially supported by the FRGS (600 RMI/FRGS 5/3 2012) UiTM grants. 
Special thanks to the National Space Agency and the National Space Centre for giving us a site to set up this project and support this project. Solar burst monitoring is a project of cooperation between the Institute of Astronomy, ETH Zurich, and FHNW Windisch, Switzerland, MARA University of Technology and University of Malaya. This paper also used NOAA Space Weather Prediction Centre (SWPC) for the sunspot, radio flux and solar flare data for comparison purpose. The research has made use of the National Space Centre Facility and a part of an initiative of the International Space Weather Initiative (ISWI) program.

\section{Biography}

Dr Zety Sharizat Hamidi is currently a senior lecturer and focused in Solar Astrophysics research specifically in radio astrophysics at the School of Physics and Material Sciences, Faculty of Sciences, MARA University of Technology, 40450, Shah Alam, Selangor, Malaysia. Involve a project under the International Space Weather Initiative (ISWI) and also a lecturer in School of Physics and Material Science, at MARA University of Technology, Shah Alam Selangor.

C. Monstein is a senior Engineer at Institute of Astronomy, Wolfgang-Pauli-Strasse 27, Building HIT, Floor J, CH-8093 Zurich, Switzerland and one of the researchers who initiated the CALLISTO system around the world.

Dr Nur Nafhatun Md Shariff is a senior lecturer in Academy of Contemporary Islamic Studies (ACIS), MARA University of Technology, 40450, Shah Alam, Selangor, Malaysia.Her current research is more on sustainability; environmental aspect. She is looking forward for cross-field research, i.e. solar astrophysics, light pollution measurement (mapping) and religious studies.

\section{References}

[1] M.R. Kundu, Solar Radio Astronomy, John Wiley, 1965.

[2] V.V. Zheleznyakov, Radio Emission of the Sun and Planets (1970).

[3] Z. Hamidi, N. Shariff, C. Monstein, First Light Detection of A Single Solar Radio Burst Type III Due To Solar Flare Event, 2014.

[4] Z. Hamidi, N. Shariff, C. Monstein, The Tendencies and Timeline of the Solar Burst Type II Fragmented, (2014).

[5] D. Herdiwijaya, The characteristics of solar wind parameters during minimum periods of solar cycle 24 and impact on Geoeffectiveness, ICAMP 2011, AIP Conf. Proc., Bandung Indonesia, 2011, pp. 4.

[6] Z. Hamidi, N. Shariff, C. Monstein, International Letters of Chemistry, Physics and Astronomy 10 (2014) 81-90.

[7] Z. Hamidi, N. Shariff, C. Monstein, W.W. Zulkifli, M. Ibrahim, N. Arifin, N. Amran, International Letters of Chemistry, Physics and Astronomy 9 (2014) 8-15.

[8] Z. Hamidi, N. Shariff, M. Ali, C. Monstein, W.W. Zulkifli, M. Ibrahim, N. Arifin, N. Amran, International Letters of Chemistry, Physics and Astronomy 9 (2014) 84-92.

[9] Z.S. Hamidi, N. Anim, N.N.M. Shariff, Z.Z. Abidin, Z.A. Ibrahim, C. Monstein, Dynamical structure of solar radio burst type III as evidence of energy of solar flares, in: R.Shukor (Ed.), PERFIK 2012, American Institute of Physics, Malaysia, 2013, pp. 11-15.

[10] N. Hashim, Z. Abidin, U. Ibrahim, R. Umar, M. Hassan, Z. Rosli, Z. Hamidi, Z. Ibrahim, Radio Astronomy in Malaysia: Current Status and Outreach Activities, Astronomical Society of the Pacific Conference Series, 2011, pp. 355. 
[11] Z. Hamidi, N. Shariff, C. Monstein, International Letters of Natural Sciences 8(1) (2014) 9-16.

[12] Z. Hamidi, N. Shariff, International Letters of Chemistry, Physics and Astronomy 5 (2014) 32-42.

[13] R. Payne-Scott, D.E. Yabsley, J.G. Bolton, Nature 160 (1947) 256-257.

[14] Boischot A., C. R. Acad. Sci. 244 (1957) 1326.

[15] N. Gopalswamy, E. Aguilar-Rodriguez, S. Yashiro, S. Nunes, M.L. Kaiser, R.A. Howard, Type II radio bursts and energetic solar eruptions, Journal of Geophysical Research 110 (2005).

[16] N. Gopalswamy, S. Akiyama, S. Yashiro, Major solar flares without coronal mass ejections, in: N. Gopalswamy, D.F. Webb (Eds.), Universal Heliophysical Processes, 2009a, pp. 283-286.

[17] Z. Hamidia, Z. Abidina, Z. Ibrahima, N. Shariffa, C. Monsteinc, Observations of Coronal Mass Ejections (CMEs) at Low Frequency Radio Region on 15th April 2012, AIP Conf. Proc 1528 (2013) 55-60.

[18] Z. Hamidi, C. Monstein, N. Shariff, Radio Observation of Coronal Mass Ejections (CMEs) Due to Flare Related Phenomenon on 7 th March 2012, (2012).

[19] N. Gopalswamy, N. Nitta, P.K. Manoharan, A. Raoult, M. Pick, Astronomy and Astrophysics 347 (1999) 684-695.

[20] N. Gopalswamy, H. Xie, S. Yashiro, I.G. and Usoskin, Coronal mass ejections and ground level enhancements, in: B.e.a. Sripathi Acharya (Ed.), 29th International Cosmic Ray Conference, Pune, India, 2005, pp. 169-172.

[21] J.P. Wild, Smerd S.F., and Weiss, A.A., Ann. Rev. Astron. Astrophysics 1 (1963) 291-366.

[22] G. Mann, Coronal Magnetic Energy Releases, Springer, Berlin, 1995.

[23] G. Mann, A. Klassen, Astron. Astrophys. 441 (2005) 319-326.

[24] N. Gopalswamy, A. Lara, M.L. Kaiser, J.L. Bougeret, J. Geophys. Res. 106 (2001) 25261-25278.

[25] N. Gopalswamy, S. Yashiro, M.L. Kaiser, R.A. Howard, J.-L. Bougeret, Journal of Geophysical Research: Space Physics 106 (2001) 29219-29229.

[26] N. Gopalswamy, A. Lara, M.L. Kaiser, J.-L. Bougeret, Journal of Geophysical Research: Space Physics 106 (2002) 25261-25277.

[27] Z. Hamidi, N. Shariff, C. Monstein, Z. Ibrahim, International Letters of Chemistry, Physics and Astronomy 7 (2014) 37-44.

[28] R. Umar, Z.Z. Abidin, Z.A. Ibrahim, M.S.R. Hassan, Z. Rosli, Z.S. Hamidi, Population density effect on radio frequencies interference (RFI) in radio astronomy, AIP Conference Proceedings 1454 (2012) 39.

[29] Z. Hamidi, N. Shariff, C. Monstein, Evaluation of Spectral Overview and Radio Frequency Interference (RFI) Sources at Four Different Sites in CALLISTO Network at the Narrow Band Solar Monitoring Region, (2014). 
[30] Z.S.Hamidi et al., International Journal of Fundamental Physical Sciences 2 (2012) 4.

[31] Z. Hamidi, N. Shariff, C. Monstein, The Different Between the Temperature of the Solar Burst at the Feed Point of the Log Periodic Dipole Antenna (LPDA) and the CALLISTO Spectrometer, (2014).

[32] Z. Hamidi, N. Shariff, International Letters of Chemistry, Physics and Astronomy 7 (2014) 30-36.

[33] R. Umar, Z. Abidin, Z. Ibrahim, N. Gasiprong, K. Asanok, S. Nammahachak, S. Aukkaravittayapun, P. Somboopon, A Prasit, N. Prasert, Z. Hamidi, N. Hashim, U.F.S.U. Ibrahim, Middle East Journal of Scientific Research 14 (2013) 861-866.

[34] Z. Hamidi, N. Shariff, C. Monstein, W.W. Zulkifli, M. Ibrahim, N. Arifin, N. Amran, International Letters of Natural Sciences (2014). 5 (2014) 10-17.

[35] Z.S.Hamidi, Z. Abidin, Z. Ibrahim, C. Monstein, N. Shariff, International Journal of Fundamental Physical Sciences 2 (2012) 32-34.

[36] Z. Hamidi, N. Shariff, Evaluation of Signal to Noise Ratio (SNR) of Log Periodic Dipole Antenna (LPDA) BEIAC, 2013.

[37] Z.S.Hamidi et al., American Journal of Modern Physics 2 (2013) 4.

[38] Z.S.Hamidi, Z. Abidin, Z. Ibrahim, N. Shariff, Indication of radio frequency interference (RFI) sources for solar burst monitoring in Malaysia, ICPAP 2011, AIP Publisher, Indonesia, 2012, pp. 6.

[39] Z. Hamidi, N. Shariff, C. Monstein, International Letters of Chemistry, Physics and Astronomy 10 (2014) 38-45.

[40] N. Anim, Z. Hamidi, Z. Abidin, C. Monstein, N. Rohizat, Radio frequency interference affecting type III solar burst observations, 2012 NATIONAL PHYSICS CONFERENCE: (PERFIK 2012), American Institute of Physics, 2013, pp. 82-86.

[41] Z.S. Hamidi, Z.Z. Abidin, Z.A. Ibrahim, N.N.M. Shariff, U.F.S.U. Ibrahim, R. Umar, Preliminary analysis of investigation Radio Frequency Interference (RFI) profile analysis at Universiti Teknologi MARA, IEEE, 2011, pp. 311-313.

[42] Z.S. Hamidi, N. Shariff, Z. Abidin, Z. Ibrahim, C. Monstein, Middle-East Journal of Scientific Research 12 (2012) 6.

[43] Z. Hamidi, N. Shariff, F.Z. Ulum, Z. Abidin, Z. Ibrahim, International Journal of Astronomy 1 (2012) 101-104.

[44] Z. S. Hamidi, N. N. M. Shariff, International Letters of Chemistry, Physics and Astronomy 4 (2014) 29-36.

[45] Z. S. Hamidi, N. N. M. Shariff, International Letters of Chemistry, Physics and Astronomy 5 (2014) 43-49.

[46] Z. S. Hamidi, N. N. M. Shariff, International Letters of Chemistry, Physics and Astronomy 7 (2014) 21-29.

[47] Z. S. Hamidi, N. N. M. Shariff, C. Monstein, W. N. A. Wan Zulkifli, M. B. Ibrahim, N. S. Arifin, N. A. Amran, International Letters of Chemistry, Physics and Astronomy 8 (2014) 13-19. 
[48] Z. S. Hamidi, N. N. M. Shariff, C. Monstein, International Letters of Chemistry, Physics and Astronomy 11(1) (2014) 51-58.

[49] Z. S. Hamidi, N. N. M. Shariff, C. Monstein, International Letters of Chemistry, Physics and Astronomy 11(2) (2014) 135-145.

[50] Z. S. Hamidi, N. N. M. Shariff, C. Monstein, International Letters of Chemistry, Physics and Astronomy 11(2) (2014) 167-176.

[51] Z. S. Hamidi, C. Monstein, N. N. M. Shariff, International Letters of Chemistry, Physics and Astronomy 11(3) (2014) 243-256. 